\title{
Neoadjuvant Chemotherapy in Operable Breast Cancer: The Pros
}

\author{
Eleftherios P. Mamounas \\ Department of Surgery, Northeastern Ohio Universities College of Medicine, Rootstown, $\mathrm{OH}$ and Cancer Center, \\ Aultman Health Foundation, Canton, $\mathrm{OH}$, USA
}

The rationale for evaluating preoperative or neoadjuvant chemotherapy in patients with operable breast cancer originated from experimental and clinical observations as well as theoretical hypotheses on tumor cell growth and dissemination [1-7]. Further clinical justification for the use of neoadjuvant chemotherapy in this setting was provided by the demonstration of equivalent survival between breast conserving surgery and mastectomy in patients with early breast cancer [8-12] and by the demonstration of significant improvements in outcome with adjuvant chemotherapy not only in patients with positive nodes but also in those with negative nodes [13, 14]. Based on these developments, neoadjuvant chemotherapy could be employed irrespective of nodal status with the intent to convert patients needing mastectomy to candidates for breast-conserving surgery.

Initial non-randomized studies of neoadjuvant chemotherapy demonstrated high rates of clinical tumor response, low rates of pathologic complete response (pCR) and increase in the rates of breast-conserving surgery [15-21]. However, non-randomized studies could not address the relative efficacy of neoadjuvant vs. adjuvant chemotherapy on disease-free and overall survival. Similarly, early randomized trials [22-26] were not designed as straightforward comparisons of neoadjuvant vs. adjuvant chemotherapy. As a result, those trials could also not adequately address the relative efficacy of each approach on outcome. This question was eventually addressed in large randomized trials that directly compared the two approaches (either with an anthracycline-based regimen or with an anthracycline-taxane-based regimen) [27-31]. These trials demonstrated no significant differences in outcome with neoadjuvant vs. adjuvant chemotherapy and, in addition, confirmed observations from previous studies i.e. that neoadjuvant chemotherapy significantly increased the rate of breast conserving surgery over mastectomy and that there was a significant correlation between achievement of $\mathrm{pCR}$ in the breast and axillary nodes and improved long-term outcome. Based on these results, neoadjuvant chemotherapy became a reasonable alternative to adjuvant chemotherapy in patients with operable breast cancer with several potential advantages over the adjuvant approach.

As mentioned above, the potential for increasing the rates of breast-conserving surgery in patients with operable breast cancer is probably the most important clinical advantage of neoadjuvant vs. adjuvant chemotherapy. It is reassuring that the increase in rate of breast conserving surgery has been achieved without a significant increase in the rate of ipsilateral breast tumor recurrence. Although it was hypothesized that the development of more effective chemotherapeutic regimens (with the incorporation of taxanes) will further increase the rates of breast conserving surgery, this hypothesis was not supported by recently reported results from a large NSABP randomized trial evaluating taxane-based chemotherapy [32]. However, besides converting patients from mastectomy to breast conserving surgery, the use of neoadjuvant chemotherapy has the potential to improve the cosmetic result by decreasing the amount of breast tissue that needs to be removed at the time of lumpectomy. This endpoint, however, is subjective and difficult to quantify and requires an accurate assessment of the patterns of primary tumor shrinkage and the amount of residual disease in the breast following neoadjuvant chemotherapy.

Besides its effects on the primary breast tumor, neoadjuvant chemotherapy has also been shown to downstage axillary lymph nodes in a significant proportion of patients (around $20-30 \%$ with anthracycline-containing regimens $[27,30]$ and close to $40 \%$ when a taxane is added $[32,33])$. Although this observation was of little clinical significance when axillary node dissection was the sole method for staging the axilla, the development and validation of sentinel node biopsy provided yet another potential advantage for the use of neoadjuvant vs.

\begin{tabular}{|c|c|c|}
\hline KARGER & (c) 2006 S. Karger GmbH, Freiburg & $\begin{array}{l}\text { Eleftherios P. Mamounas, M.D., M.P.H., F.A.C.S. } \\
\text { Cancer Center }\end{array}$ \\
\hline $\begin{array}{l}\text { Fax }+497614520714 \\
\text { E-mail Information@Karger.de } \\
\text { www.karger.com }\end{array}$ & $\begin{array}{l}\text { Accessible online at: } \\
\text { www.karger.com/brc }\end{array}$ & $\begin{array}{l}\text { Aultman Health Foundation } \\
2600 \text { 6th Street, Canton, OH 44710, USA } \\
\text { Tel. +1 330-438 6281, Fax -363 7367 } \\
\text { E-mail tmamounas@aultman.com }\end{array}$ \\
\hline
\end{tabular}


adjuvant chemotherapy. Thus, in patients with involved axillary nodes at presentation, down-staging of the nodes with neoadjuvant chemotherapy and performance of sentinel node biopsy instead of axillary dissection could lead to a significant decrease in the extent and morbidity of axillary surgery. This approach is, naturally, predicated on the premise that sentinel node biopsy is feasible and accurate following neoadjuvant chemotherapy. Until recently, only small, single-institution studies have examined the efficacy of lymphatic mapping and the accuracy of sentinel node biopsy after neoadjuvant chemotherapy [34-46]. Because of the small number of patients (and, more importantly, small number of node positive patients) in those studies, significant variability was observed in the rate of sentinel node identification and, more importantly, in the rate of false negative sentinel nodes. However, when these studies are examined collectively or when larger, multi-center data sets are analyzed [47, 48], sentinel node biopsy after neoadjuvant chemotherapy appears to have similar performance characteristics to those of sentinel node biopsy before systemic therapy [49-52].

Following neoadjuvant chemotherapy, the resulting separation of patients into different outcome groups according to the achievement or not achievement of pathologic complete response may provide a significant clinical advantage in patient management. Since this association appears to be maintained with the use of more effective chemotherapy regimens [31, 53, 54], pCR can be used as an intermediate endpoint in determining the value of new chemotherapy regimens or new drugs administered after well-established regimens [55]. From a research standpoint, because this intermediate endpoint can be achieved within weeks from the start of neoadjuvant chemotherapy, the relative efficacy of new regimens can be evaluated promptly and useful information can be obtained without a long waiting follow up period, as is currently the case with the use of adjuvant chemotherapy. From a clinical practice standpoint, by evaluating clinical response to neoadjuvant chemotherapy (a surrogate to $\mathrm{pCR}$ ) individual patients might be spared from repeated administration of ineffective regimens if these do not translate into prompt tumor response. In the opposite end, one of the original premises for the use of neoadjuvant chemotherapy was that clinical breast tumor response could be used as an in vivo chemosensitivity assay in order to predict which patients would or would not benefit from additional (potentially non-cross-resistant) chemotherapy.
Although the existing information on the subject is limited, the preliminary conclusions seem to be somewhat counterintuitive to the original hypothesis, i.e. that patients who do not respond to the initial neoadjuvant chemotherapy regimen might derive the most benefit from subsequent non-cross resistant chemotherapy. In fact, the results so far point to the opposite conclusion - that patients who show a clinical response to the first chemotherapy regimen are those who derive the most benefit from non-cross resistant chemotherapy $[53,56,57]$ and those who do not show a clinical response to the first regimen have relatively chemo-resistant disease and derive little, if any, benefit from non-cross resistant regimens $[53,58]$.

Finally, another advantage of neoadjuvant chemotherapy is the potential for evaluation of many of the proven, as well as putative, prognostic tumor markers and their correlation with clinical and pathologic tumor response and eventually outcome [55]. Furthermore, by serially monitoring biomarker changes during and after neoadjuvant chemotherapy, one could gain biologic insight into the nature and function of these biomarkers (as they relate to chemosensitivity or chemoresistance) and into the mechanism(s) of action of novel chemotherapeutic regimens or new treatment modalities. As we rapidly enter the era of gene expression profiling through the use of high-throughput technology and other novel molecular techniques, clinical and, more importantly, pathologic response to neoadjuvant chemotherapy are useful endpoints for identifying gene profiles that predict for response to a certain chemotherapy agent or particular drug combination [59-63]. This approach has the potential to rapidly optimize the selection of appropriate candidates for particular neoadjuvant therapy regimens avoiding unnecessary toxicity in patients who are unlikely to benefit. Although such an approach can be also utilized in the adjuvant setting, the neoadjuvant setting can provide the necessary information in a much more expeditious fashion allowing us to quickly improve our understanding of tumor biology and individualize treatment options for our patients.

In summary, several advantages exist with the use of neoadjuvant chemotherapy in patients with operable breast cancer. These advantages have significant clinical and biologic implications and will allow us to rapidly evolve our understanding of the disease and to continue to expand the available treatment options for our patients.

\section{References}

1 Perloff M, Lesnick GJ: Chemotherapy before and after mastectomy in stage III breast cancer. Arch Surg 1882;117:879-81.

2 Schick P, Goodstein J, Moor J, et al.: Preoperative chemotherapy followed by mastectomy for locally advanced breast cancer. J Surg Oncol 1983;22: 278-82.
3 Sorace RA, Bagley CS, Lichter AS, et al.: The management of nonmetastatic locally advanced breast cancer using primary induction chemotherapy with hormonal synchronization followed by radiation therapy with or without debulking surgery. World J Surg 1985;9:775-85.
4 Skipper HE: Kinetics of mammary tumor cell growth and implications for therapy. Cancer 1971; 28:1479-99.

$\checkmark 5$ Goldie JH, Coldman AJ: A mathematic model for relating the drug sensitivity of tumors to their spontaneous mutation rate. Cancer Treat Rep 1979;63: 1727-33 
6 Gunduz N, Fisher B, Saffer EA: Effect of surgical removal on the growth and kinetics of residual tumor. Cancer Res 1979;39:3861-5.

7 Fisher B, Gunduz N, Saffer EA: Influence of the interval between primary tumor removal and chemotherapy on kinetics and growth of metastases. Cancer Res 1983;43:1488-92.

8 Fisher B, Redmond C, Poisson R, et al.: Eight-year results of a randomized clinical trial comparing total mastectomy and lumpectomy with or without irradiation in the treatment of breast cancer. N Engl J Med 1989;320:822-8.

$\checkmark 9$ Fisher B, Anderson S, Redmond CK, et al.: Reanalysis and results after 12 years of follow-up in a randomized clinical trial comparing total mastectomy with lumpectomy with or without irradiation in the treatment of breast cancer. N Engl J Med 1995; 333:1456-61.

10 Veronesi U, Saccozzi R, Del Vecchio M, et al.: Comparing radical mastectomy with quadrantectomy, axillary dissection, and radiotherapy in patients with small cancers of the breast. N Engl J Med 1981;305:6-11.

11 Fisher B, Anderson S, Bryant J, et al.: Twenty-year follow-up of a randomized trial comparing total mastectomy, lumpectomy, and lumpectomy plus irradiation for the treatment of invasive breast cancer. N Engl J Med 2002;347:1233-41.

12 Veronesi U, Cascinelli N, Mariani L, et al.: Twentyyear follow-up of a randomized study comparing breast-conserving surgery with radical mastectomy for early breast cancer. N Engl J Med 2002;347: 1227-32.

13 Group EBCTC: Systemic treatment of early breast cancer by hormonal, cytotoxic, or immune therapy. 133 randomised trials involving 31,000 recurrences and 24,000 deaths among 75,000 women. Early Breast Cancer Trialists' Collaborative Group. Lancet 1992;339:71-85.

14 Group EBCTC: Polychemotherapy for early breast cancer: an overview of the randomised trials. Early Breast Cancer Trialists' Collaborative Group. Lancet 1998;352:930-42.

15 Jacquillat C, Weil M, Baillet F, et al.: Results of neoadjuvant chemotherapy and radiation therapy in the breast-conserving treatment of 250 patients with all stages of infiltrative breast cancer. Cancer 1990;66:119-29.

16 Bonadonna G, Veronesi U, Brambilla C, et al.: Primary chemotherapy to avoid mastectomy in tumors with diameters of three centimeters or more. J Natl Cancer Inst 1990;82:1539-45.

17 Tubiana-Hulin M, Malek M, Brifford M, et al.: Preoperative chemotherapy of operable breast cancer (stage IIIA). Prognostic factors of distant recurrence. Eur J Cancer 1993;29A:S76 (\#391).

-18 Belembaogo E, Feillel V, Chollet P, et al.: Neoadjuvant chemotherapy in 126 operable breast cancers. Eur J Cancer 1992;28A:896-900.

19 Smith IE, Jones AL, O'Brien ME, et al.: Primary medical (neo-adjuvant) chemotherapy for operable breast cancer. Eur J Cancer 1993;29A:1796-9.

20 Chollet P, Charrier S, Brain E, et al.: Clinical and pathological response to primary chemotherapy in operable breast cancer. Eur J Cancer 1997;33: 862-6.

21 Gradishar WJ: Docetaxel as neoadjuvant chemotherapy in patients with stage III breast cancer. Oncology (Huntingt) 1997;11:15-8.

22 Mauriac L, Durand M, Avril A, et al.: Effects of primary chemotherapy in conservative treatment of breast cancer patients with operable tumors larger than $3 \mathrm{~cm}$. Results of a randomized trial in a single centre. Ann Oncol 1991;2:347-54
23 Scholl SM, Fourquet A, Asselain B, et al.: Neoadjuvant versus adjuvant chemotherapy in premenopausal patients with tumours considered too large for breast conserving surgery: preliminary results of a randomised trial: S6. Eur J Cancer 1994; 30A:645-52.

24 Scholl SM, Pierga JY, Asselain B, et al.: Breast tumour response to primary chemotherapy predicts local and distant control as well as survival. Eur J Cancer 1995;31A:1969-75

25 Powles TJ, Hickish TF, Makris A, et al.: Randomized trial of chemoendocrine therapy started before or after surgery for treatment of primary breast cancer. J Clin Oncol 1995;13:547-52.

26 Makris A, Powles TJ, Ashley SE, et al.: A reduction in the requirements for mastectomy in a randomized trial of neoadjuvant chemoendocrine therapy in primary breast cancer. Ann Oncol 1998;9: 1179-84.

27 Fisher B, Brown A, Mamounas E, et al.: Effect of preoperative chemotherapy on local-regional disease in women with operable breast cancer: findings from National Surgical Adjuvant Breast and Bowel Project B-18. J Clin Oncol 1997;15:2483-93.

28 Fisher B, Bryant J, Wolmark N, et al.: Effect of preoperative chemotherapy on the outcome of women with operable breast cancer. J Clin Oncol 1998;16: 2672-85.

29 Wolmark N, Wang J, Mamounas E, et al.: Preoperative chemotherapy in patients with operable breast cancer: nine-year results from National Surgical Adjuvant Breast and Bowel Project B-18. J Natl Cancer Inst Monogr:2001;96-102.

30 van der Hage JA, van de Velde CJ, Julien JP, et al.: Preoperative chemotherapy in primary operable breast cancer: results from the European Organization for Research and Treatment of Cancer trial 10902. J Clin Oncol 2001;19:4224-37.

Gianni L, Baselga J, Eirmann W, et al.: European Cooperative Trial in Operable Breast Cancer (ECTO): Improved freedom from progression (FFP) from adding paclitaxel (T) to doxorubicin (A) followed by cyclophosphamide methotrexate and fluorouracil (CMF). J Clin Oncol 2005;23:7S, abstr 513.

32 Bear HD, Anderson S, Brown A, et al.: The effect on tumor response of adding sequential preoperative docetaxel to preoperative doxorubicin and cyclophosphamide: preliminary results from National Surgical Adjuvant Breast and Bowel Project Protocol B-27. J Clin Oncol 2003;21:4165-74.

33 Gianni L BJ, Eiermann W, et al..: First report of the European Cooperative Trial in operable breast cancer (ECTO): effect of primary systemic therapy. Proc Am Soc Clin Oncol 2002;21:34a, abstr 132.

34 Breslin TM, Cohen L, Sahin A, et al.: Sentinel lymph node biopsy is accurate after neoadjuvant chemotherapy for breast cancer. J Clin Oncol 2000; 18:3480-6.

35 Nason KS, Anderson BO, Byrd DR, et al.: Increased false negative sentinel node biopsy rates after preoperative chemotherapy for invasive breast carcinoma. Cancer 2000;89:2187-94.

36 Julian TB, Patel N, Dusi D, et al.: Sentinel lymph node biopsy after neoadjuvant chemotherapy for breast cancer. Am J Surg 2001;182:407-10.

37 Stearns V, Ewing CA, Slack R, et al.: Sentinel lymphadenectomy after neoadjuvant chemotherapy for breast cancer may reliably represent the axilla except for inflammatory breast cancer. Ann Surg Oncol 2002;9:235-42.
38 Fernandez A, Cortes M, Benito E, et al.: Gamma probe sentinel node localization and biopsy in breast cancer patients treated with a neoadjuvant chemotherapy scheme. Nucl Med Commun 2001; 22:361-6.

39 Haid A, Tausch C, Lang A, et al.: Is sentinel lymph node biopsy reliable and indicated after preoperative chemotherapy in patients with breast carcinoma? Cancer 2001:92:1080-4.

40 Miller AR, Thomason VE, Yeh IT, et al.: Analysis of sentinel lymph node mapping with immediate pathologic review in patients receiving preoperative chemotherapy for breast carcinoma. Ann Surg Oncol 2002;9:243-7.

41 Reitsamer R, Peintinger F, Rettenbacher L, et al.: Sentinel lymph node biopsy in breast cancer patients after neoadjuvant chemotherapy. J Surg Oncol 2003;84:63-7.

42 Brady EW: Sentinel lymph node mapping following neoadjuvant chemotherapy for breast cancer. Breast J 2002;8:97-100.

43 Schwartz GF, Meltzer AJ: Accuracy of axillary sentinel lymph node biopsy following neoadjuvant (induction) chemotherapy for carcinoma of the breast. Breast J 2003;9:374-9.

44 Balch GC, Mithani SK, Richards KR, et al.: Lymphatic mapping and sentinel lymphadenectomy after preoperative therapy for stage II and III breast cancer. Ann Surg Oncol 2003;10:616-21.

45 Piato JR, Barros AC, Pincerato KM, et al.: Sentinel lymph node biopsy in breast cancer after neoadjuvant chemotherapy. A pilot study. Eur J Surg Oncol 2003;29:118-20.

46 Aihara T, Munakata S, Morino H, et al.: Feasibility of sentinel node biopsy for breast cancer after neoadjuvant endocrine therapy: a pilot study. J Surg Oncol 2004;85:77-81.

47 Mamounas EP: Sentinel lymph node biopsy after neoadjuvant systemic therapy. Surg Clin North Am 2003;83:931-42.

48 Mamounas EP, Brown A, Anderson S, et al.: Sentinel node biopsy after neoadjuvant chemotherapy in breast cancer: Results from National Surgical Adjuvant Breast and Bowel Prolect Protocol B-27. J Clin Oncol 2005;23:2694-2702.

49 O'Hea BJ, Hill AD, El-Shirbiny AM, et al.: Sentinel lymph node biopsy in breast cancer: initial experience at Memorial Sloan-Kettering Cancer Center. J Am Coll Surg 1998;186:423-7.

50 McMasters KM, Tuttle TM, Carlson DJ, et al.: Sentinel lymph node biopsy for breast cancer: a suitable alternative to routine axillary dissection in multi-institutional practice when optimal technique is used. J Clin Oncol 2000;18:2560-6.

51 Krag DN, Weaver DL, Alex JC, et al.: Surgical resection and radiolocalization of the sentinel lymph node in breast cancer using a gamma probe. Surg Oncol 1993;2:335-9; discussion 340.

52 Giuliano AE, Kirgan DM, Guenther JM, et al.: Lymphatic mapping and sentinel lymphadenectomy for breast cancer. Ann Surg 1994;220:391-8; discussion 398-401.

53 Bear HD, Anderson S, Smith RE, et al.: Sequential preoperative or postoperative docetaxel added to preoperative doxorubicin plus cyclophosphamide for operable breast cancer: National Surgical Adjuvant Breast and Bowel Project Protocol B-27. J Clin Oncol 2006;24:2019-27. 
54 Buzdar AU, Ibrahim NK, Francis D, et al.: Significantly higher pathologic complete remission rate after neoadjuvant therapy with trastuzumab, paclitaxel, and epirubicin chemotherapy: results of a randomized trial in human epidermal growth factor receptor 2-positive operable breast cancer. J Clin Oncol 2005;23:3676-85.

55 Fisher B, Mamounas EP: Preoperative chemotherapy: a model for studying the biology and therapy of primary breast cancer. J Clin Oncol 1995;13:537-40.

56 Smith IC, Heys SD, Hutcheon AW, et al.: Neoadjuvant chemotherapy in breast cancer: significantly enhanced response with docetaxel. J Clin Oncol 2002;20:1456-66.
Thomas E, Holmes FA, Smith, TL, et al.: The use of alternate, non-cross-resistant adjuvant chemotherapy on the basis of pathologic response to a neoadjuvant doxorubicin-based regimen in women with operable breast cancer: long-term results from a prospective randomized trial. J Clin Oncol 2004; 22:2294-2302.

58 von Minckwitz G, Raab G, Blohmer JU, et al.: Primary chemotherapy adapted on in-vivo-chemosensitivity in patients with primary breast cancer. The pilot GEPARTRIO study. Proc Am Soc Clin Oncol 2003;22:22, abstr 85.

59 Ayers M, Symmans WF, Stec J, et al.: Gene expression profiles predict complete pathologic response to neoadjuvant paclitaxel and fluorouracil, doxorubicin, and cyclophosphamide chemotherapy in breast cancer. J Clin Oncol 2004;22:2284-93.
60 Thuerigen O, Scneeweiss A, Toedt G, et al.: Gene expression signature predicting pathologic complete response with gemcitabine, epirubicin, and docetaxel in primary breast cancer. J Clin Oncol 2006;24:1839-45.

61 Gianni L, Zambetti M, Clark K, et al.: Gene expression profiles in paraffin-embedded core biopsy tissue predict response to chemotherapy in women with locally advanced breast cancer. J Clin Oncol 2005;23:7265-77.

62 Paik S, Tang G, Shak S, et al.: Gene expression and benefit of chemotherapy in women with node-negative, estrogen receptor-positive breast cancer. J Clin Oncol 2006;24:3726-34.

63 Chang JC, Wooten EC, Tsimelzon A, et al.: Patterns of resistance and incomplete response to docetaxel by gene expression profiling in breast cancer patients. J Clin Oncol 2005;23:1169-77.

\section{Erratum}

In the article

\section{Peripheral Neuroectodermal Tumor (PNET) of the Breast - A 6-Year Follow-Up (Breast Care 2006;1:324-327, printed issue) one author was accidentally left out. The full author list must read:}

\author{
Anke Thomas $^{\mathrm{a}}$ Jens U. Blohmer ${ }^{\mathrm{b}}$ Andreas Turzynskic \\ Orhan Sezer $^{d}$ Thomas Fischere ${ }^{\mathrm{e}}$ Gundula Thiel $^{f}$ \\ Manfred Dietelg Werner Lichtenegger ${ }^{\mathrm{a}}$ Sherko Kümmel ${ }^{\mathrm{h}}$ \\ a Department of Senology, University Medicine Berlin, Charité, \\ ${ }^{b}$ Department of Senology, St. Gertrauden Hospital, \\ ${ }^{c}$ Gemeinschaftspraxis für Pathologie, Lübeck, \\ dDepartment of Medical Oncology and Hematology, \\ e Institute of Radiology, \\ f Institute for Genetics, \\ g Institute of Pathology, University Medicine Berlin, Charité, Berlin, \\ h Department of Gynecology and Obstetrics, University Essen, Germany
}

We apologize for this mistake. 